\title{
Pathogenesis of persistent lymphatic vessel hyperplasia in chronic airway inflammation
}

\author{
Peter Baluk, ${ }^{1}$ Tuomas Tammela, ${ }^{2}$ Erin Ator, ${ }^{1}$ Natalya Lyubynska, ${ }^{1}$ Marc G. Achen, ${ }^{3}$ \\ Daniel J. Hicklin, ${ }^{4}$ Michael Jeltsch, ${ }^{2}$ Tatiana V. Petrova, ${ }^{2}$ Bronislaw Pytowski, ${ }^{4}$ Steven A. Stacker, ${ }^{3}$ \\ Seppo Ylä-Herttuala, ${ }^{5}$ David G. Jackson, ${ }^{6}$ Kari Alitalo, ${ }^{2}$ and Donald M. McDonald ${ }^{1}$ \\ ${ }^{1}$ Cardiovascular Research Institute, Comprehensive Cancer Center, and Department of Anatomy, UCSF, San Francisco, California, USA. \\ ${ }^{2}$ Molecular/Cancer Biology Laboratory and Ludwig Institute for Cancer Research, University of Helsinki, Helsinki, Finland. \\ ${ }^{3}$ Ludwig Institute for Cancer Research, Royal Melbourne Hospital, Parkville, Victoria, Australia. ${ }^{4}$ mClone Systems Inc., New York, New York, USA. \\ ${ }^{5}$ A.I. Virtanen Institute and Department of Medicine, University of Kuopio, Kuopio, Finland. ${ }^{6}$ University of Oxford, Nuffield Department of Medicine/Medical \\ Research Council Human Immunology Unit, Weatherall Institute of Molecular Medicine, John Radcliffe Hospital, Headington, Oxford, United Kingdom.
}

\begin{abstract}
Edema occurs in asthma and other inflammatory diseases when the rate of plasma leakage from blood vessels exceeds the drainage through lymphatic vessels and other routes. It is unclear to what extent lymphatic vessels grow to compensate for increased leakage during inflammation and what drives the lymphangiogenesis that does occur. We addressed these issues in mouse models of (a) chronic respiratory tract infection with Mycoplasma pulmonis and (b) adenoviral transduction of airway epithelium with VEGF family growth factors. Blood vessel remodeling and lymphangiogenesis were both robust in infected airways. Inhibition of VEGFR-3 signaling completely prevented the growth of lymphatic vessels but not blood vessels. Lack of lymphatic growth exaggerated mucosal edema and reduced the hypertrophy of draining lymph nodes. Airway dendritic cells, macrophages, neutrophils, and epithelial cells expressed the VEGFR-3 ligands VEGF-C or VEGF-D. Adenoviral delivery of either VEGF-C or VEGF-D evoked lymphangiogenesis without angiogenesis, whereas adenoviral VEGF had the opposite effect. After antibiotic treatment of the infection, inflammation and remodeling of blood vessels quickly subsided, but lymphatic vessels persisted. Together, these findings suggest that when lymphangiogenesis is impaired, airway inflammation may lead to bronchial lymphedema and exaggerated airflow obstruction. Correction of defective lymphangiogenesis may benefit the treatment of asthma and other inflammatory airway diseases.
\end{abstract}

\section{Introduction}

Lymphatic vessels provide key routes for drainage of interstitial fluid from tissues. Edema, a cardinal sign of inflammation and clinically significant feature of inflammatory disease, results when the amount of leakage from inflamed blood vessels exceeds the capacity of lymphatic vessels for drainage. Despite the recognition that angiogenesis and mucosal swelling can increase airflow resistance in obstructive lung disease $(1,2)$, remarkably little is known about the status of lymphatic vessels and factors that influence fluid drainage from the respiratory tract. Lymphatic vessels may proliferate under inflammatory conditions (3), yet the time course, extent, molecular mechanisms, and functional consequences of lymphangiogenesis are largely unknown.

Vascular endothelial growth factors VEGF-C and VEGF-D drive lymphangiogenesis via VEGFR-3 signaling (4-7). The exclusivity of this effect is unclear, because VEGF-C and VEGF-D may also induce angiogenesis $(6,8,9)$. Added to the list of potential growth factors, VEGF (VEGF-A, also known as vascular permeability factor [VPF]), well known for its potent angiogenic actions, and PDGF appear to drive lymphatic growth under certain conditions $(10,11)$.

Nonstandard abbreviations used: VEGF-D $\Delta \mathrm{N} \Delta \mathrm{C}$, processed VEGF-D truncated at $\mathrm{N}$ and $\mathrm{C}$ termini.

Conflict of interest: D.J. Hicklin and B. Pytowski are employees of ImClone Systems Inc. and hold stock or stock options in the company. M.G. Achen owns shares in Lymphatix Ltd., which has an interest in the field of research described in this work.

Citation for this article: J. Clin. Invest. 115:247-257 (2005)

doi:10.1172/JCI200522037.
Impairment of lymphatic drainage, most commonly caused by genetic mutation, filarial infection, surgery, or trauma, results in lymphedema with sometimes marked swelling of limbs or other regions (12). It follows that defects in lymphangiogenesis in the respiratory tract, especially when plasma leakage is exaggerated by inflammation, may produce edema of the airway mucosa (bronchial lymphedema). Because of the strategic role of mucosal thickness in determining the effect of bronchial constriction on airway caliber and airflow resistance (1), bronchial lymphedema could exacerbate airway obstruction in inflammatory disease.

Blood vessels that proliferate and undergo remodeling in inflammatory airway disease rapidly return to normal as the inflammation resolves during treatment $(13,14)$. However, lymphatic growth may not be so readily reversible $(15,16)$. Persistence of new lymphatic networks after resolution of inflammation would leave in place a drainage system for fluid and immune cells that may accelerate responses in subsequent inflammatory episodes.

The present study exploited 2 novel and versatile mouse models of lymphangiogenesis to develop a better understanding of airway lymphatic vessels in health and disease. Here, we show that Mycoplasma pulmonis infection, which causes inflammatory cell influx, angiogenesis, mucosal edema, epithelial changes, and fibrosis typical of chronic airway inflammation (17), also induces robust lymphangiogenesis. Inhibition of VEGFR-3 signaling entirely blocked sprouting of lymphatic vessels but had no effect on angiogenesis. Similarly, adenoviral transduction of airway epithelium with VEGFR-3 ligands VEGF-C or VEGF-D induced lymphangiogenesis similar to that in $M$. pulmonis infection but no angiogenesis. Conversely, adenoviral delivery 

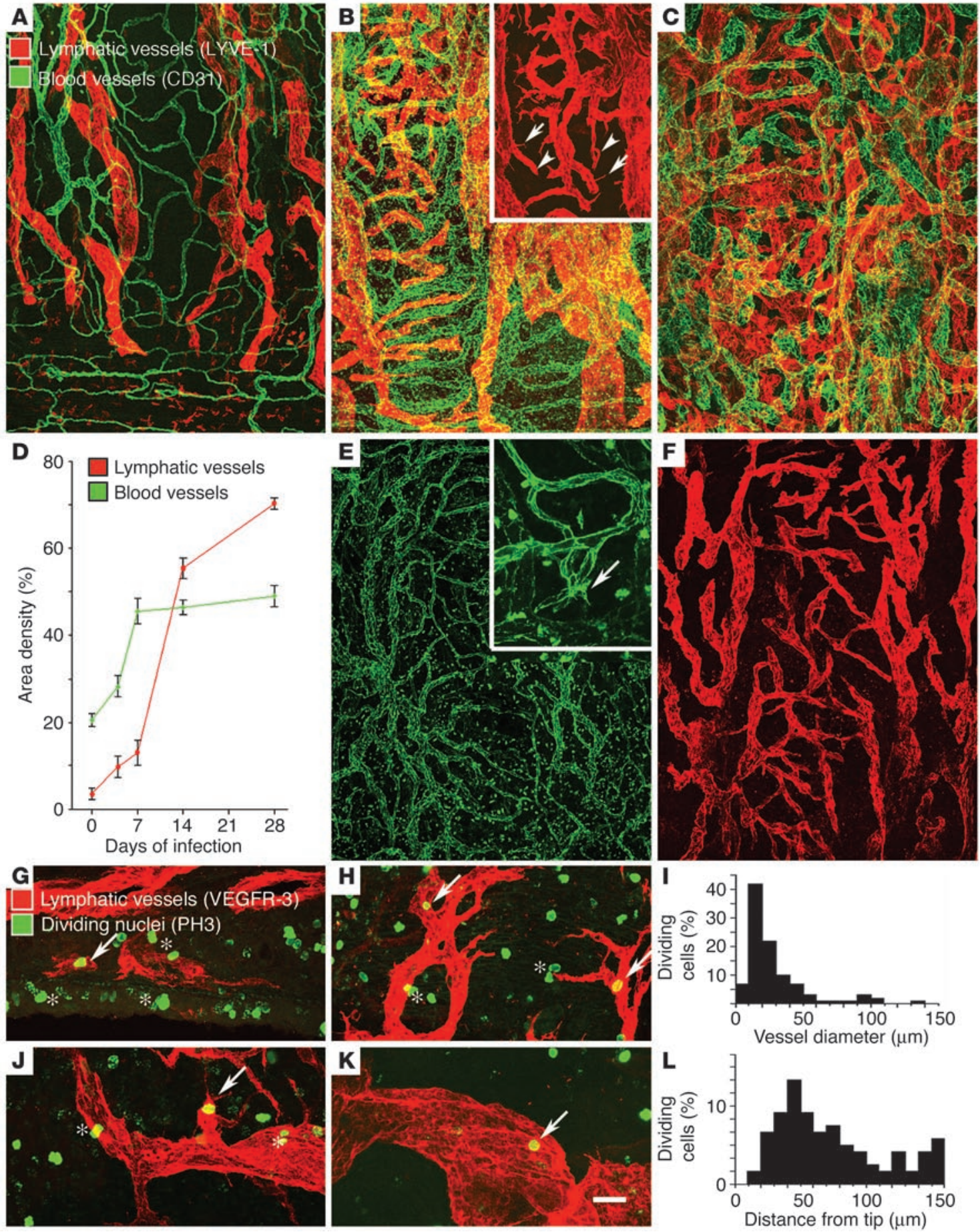

\section{Figure 1}

Lymphangiogenesis and angiogenesis after M. pulmonis infection. (A-C, E, and F) Confocal micrographs of tracheal whole mounts stained for lymphatic vessels (red) and blood vessels (green). (A) Pathogen-free C3H mouse. (B) C3H mouse infected for 14 days; inset shows lymphatic sprouts (arrowheads) and filopodia (arrows). (C) C3H mouse infected for 28 days. (D) Proliferation of lymphatic vessels (red) and blood vessels (green) in tracheas of C3H mice over 28 days of infection. (E) In a C57BL/6 mouse infected for 14 days, blood vessels (CD31) exhibit sprouting (arrow) and enlargement. (F) Same region as shown in E. Lymphatic vessels (LYVE-1) have a growth pattern similar to that of C3H mice. (G, H, J, and $\mathbf{K}$ ) Dividing endothelial cells stained for phosphohistone $\mathrm{H} 3$ (PH3, green) in tracheal lymphatic vessels (red) of C3H mice infected for 14 days. (G) Section of trachea showing dividing cells, which are sparse in lymphatic vessels (arrow) and numerous in epithelial cells and leukocytes (asterisks). (H, J, and K) Tracheal whole mounts. (H) Dividing lymphatic endothelial cells (arrows) in stalks of mediumsized sprouts. (I) Size distribution of 100 dividing lymphatic endothelial cells. Most dividing cells are near sprout tips (J) or in larger lymphatic vessels (K). (L) Distribution of distances of dividing lymphatic endothelial cells from sprout tips. Scale bar in $\mathbf{K}$ applies to all figures: $100 \mu \mathrm{m}$ in $\mathbf{A}-\mathbf{C}, \mathbf{E}$ and $\mathbf{F}$, and 20 um in $\mathbf{G}, \mathbf{H}, \mathbf{J}$, and $\mathbf{K}$. 
of VEGF induced angiogenesis but not lymphangiogenesis. Inflammatory cells and some resident cells were identified as cellular sources of VEGF-C or VEGF-D in infected airways. Studies of the functional consequences of blocking lymphangiogenesis revealed that inhibition of VEGFR-3 signaling in M. pulmonis-infected mice exaggerated mucosal edema and reduced lymph node hypertrophy. Comparison of the reversibility of angiogenesis and lymphangiogenesis after antibiotic treatment showed that inflammation and blood vessel remodeling were largely resolved within 2 weeks, but an extensive network of new lymphatic vessels remained in the airway mucosa even after 12 weeks of treatment.

\section{Results}

Blood and lymphatic vasculature of normal airways. In the airways of pathogen-free mice, blood vessels and lymphatic vessels formed discrete, segmented networks aligned with the framework of cartilage rings (Figure 1A). Most arterioles and venules, identified by CD31 immunoreactivity, were located in the tissue between cartilage rings, whereas most blood capillaries were in regions of mucosa overlying rings. Lymphatic vessels, identified as blind-ended, LYVE-1-immunoreactive vessels approximately $70 \mu \mathrm{m}$ in diameter on average, were restricted to the mucosa between rings. Lymphatic vessels also showed CD31 immunoreactivity, but this was much weaker than in blood vessels.

Both blood vessels and lymphatic vessels grow in airway inflammation. During the first few days after $M$. pulmonis infection, mucosal blood vessels enlarged conspicuously after endothelial cell proliferation (18), but no changes were evident in lymphatic vessels. Blood vessel growth reached a plateau at 14 days (Figure 1B). The earliest signs of lymphatic growth were detected at 7 days, when numerous delicate filopodia and larger tubular sprouts were visible along lymphatic vessels (Figure 1B, inset). Lymphatic sprouts were most abundant on the surface facing cartilage rings and oriented toward the overlying airway epithelium. During the second week, lymphatic sprouts enlarged and fused to form a 3-dimensional network (Figure 1, $\mathrm{B}$ and $\mathrm{C}$ ). Although blood vascular and lymphatic networks were intimately intertwined, no connections between the 2 systems were detected. Measurements confirmed that blood vessel growth was greatest during the first week, but the rate of lymphatic growth peaked in the second week, and thereafter lymphatic vessels were more abundant than blood vessels (Figure 1D).

Growth of airway lymphatic vessels after infection was similar in $\mathrm{C} 3 \mathrm{H}$ mice and $\mathrm{C} 57 \mathrm{BL} / 6$ mice. However, remodeling of mucosal blood vessels differed in the

Figure 2
2 strains (19). Vascular enlargement was most conspicuous in $\mathrm{C} 3 \mathrm{H}$ mice, but enlargement was accompanied by extensive proliferation in C57BL/6 mice (Figure 1, E and F). In both strains, vascular remodeling resulted in the transformation of capillaries into venules that supported abundant leukocyte adhesion.

Endothelial cells of growing blood vessels divide at the base of sprouts, advancing the filopodia-rich but nondividing tip cells (20). To determine the location of dividing endothelial cells in growing lymphatic vessels, we stained tracheal whole mounts for phosphohistone $\mathrm{H} 3$, a marker of M-phase of cell division (20). Most dividing LYVE-1-positive endothelial cells were located in small lymphatic vessels (with a median diameter $22 \mu \mathrm{m}$ ), not at sprout tips (Figure $1, \mathrm{G}-\mathrm{I})$. The separation between dividing lymphatic endothelial cells and sprout tips was on average $63 \mu \mathrm{m}$ (Figure 1L). Only 10\% of
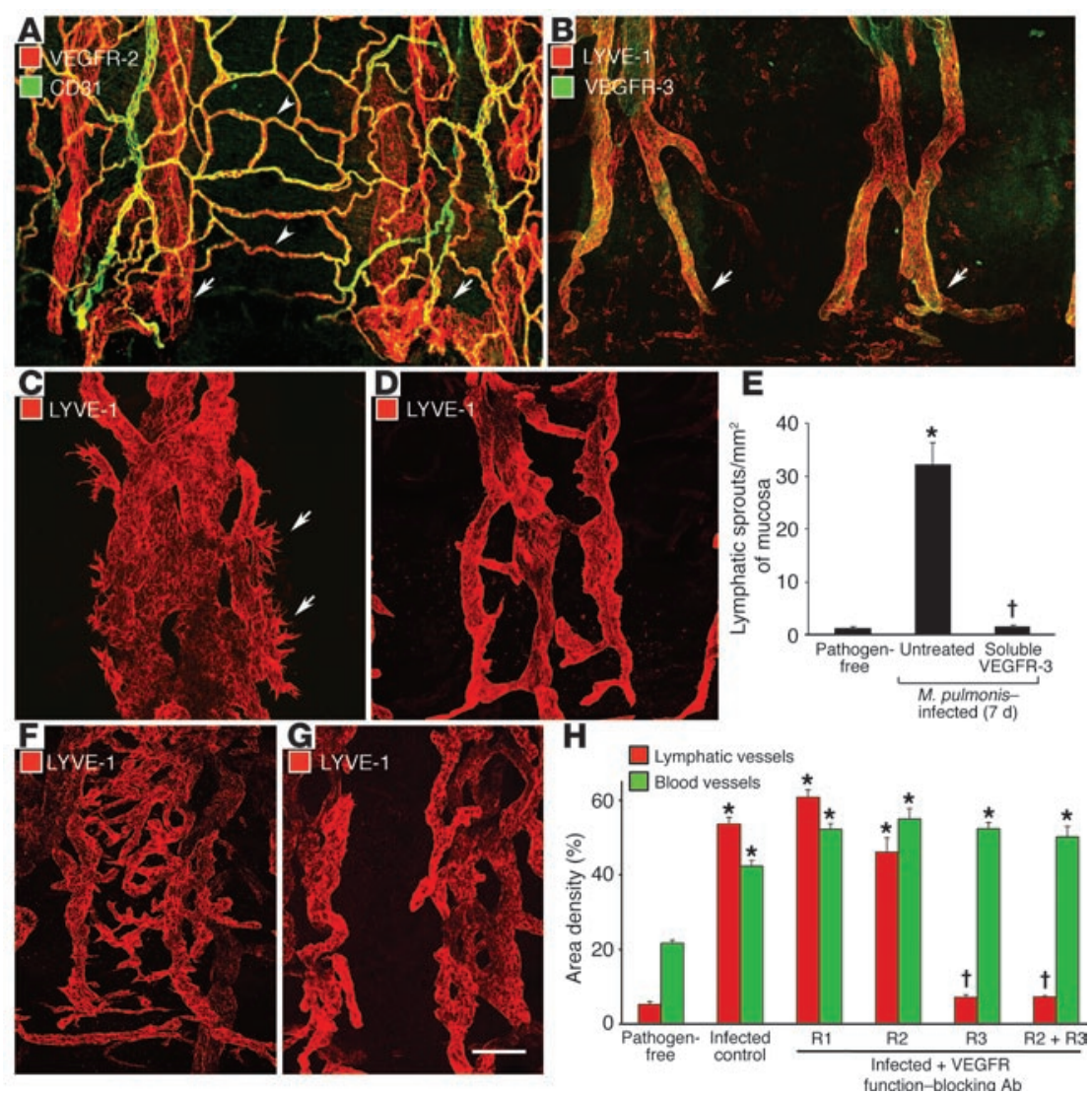

VEGF receptor distribution and inhibition in tracheal vessels. (A) Strong VEGFR-2 immunoreactivity on blood vessels (arrowheads) and weaker staining of lymphatic vessels (arrows) in pathogen-free mouse. (B) Immunoreactivities for VEGFR-3 and LYVE-1 colocalize on lymphatic vessels (arrows) in pathogen-free mouse. (C and D) Lymphatic vessels in mice infected with $M$. pulmonis for 7 days, either untreated $(\mathbf{C})$ or pretreated with soluble VEGFR-3-Ig (D). Sprouts (arrows) emerge from enlarged lymphatic vessels after infection (C) but not if pretreated with soluble VEGFR-3-Ig (D). (E) Number of lymphatic sprouts in tracheas from pathogen-free mice, or from mice after 7 days of infection, with or without soluble VEGFR-3-Ig pretreatment. (F and G) After 14 days of infection, lymphangiogenesis is not affected by concurrent treatment with antibody against VEGFR-1 (F) but is blocked by antibody against VEGFR-3 (G). (H) Area density of lymphatic vessels (red) and blood vessels (green) showing inhibition of infection-induced lymphangiogenesis by anti-VEGFR-3 (R3) antibody but not by anti-VEGFR-1 (R1) or anti-VEGFR-2 (R2). Anti-VEGFR-2 did not augment the effect of anti-VEGFR-3 (R2 + R3). None of the antibodies reduced angiogenesis. ${ }^{\star} P<0.05$ vs. pathogen-free group; ${ }^{\dagger} P<0.05$ vs. infected control group. Scale bar in $\mathbf{G}$ applies to all figures: $100 \mu \mathrm{m}$ in $\mathbf{A}$ and $\mathbf{B}$ and $50 \mu \mathrm{m}$ in $\mathbf{C}, \mathbf{D}, \mathbf{F}$, and $\mathbf{G}$. 

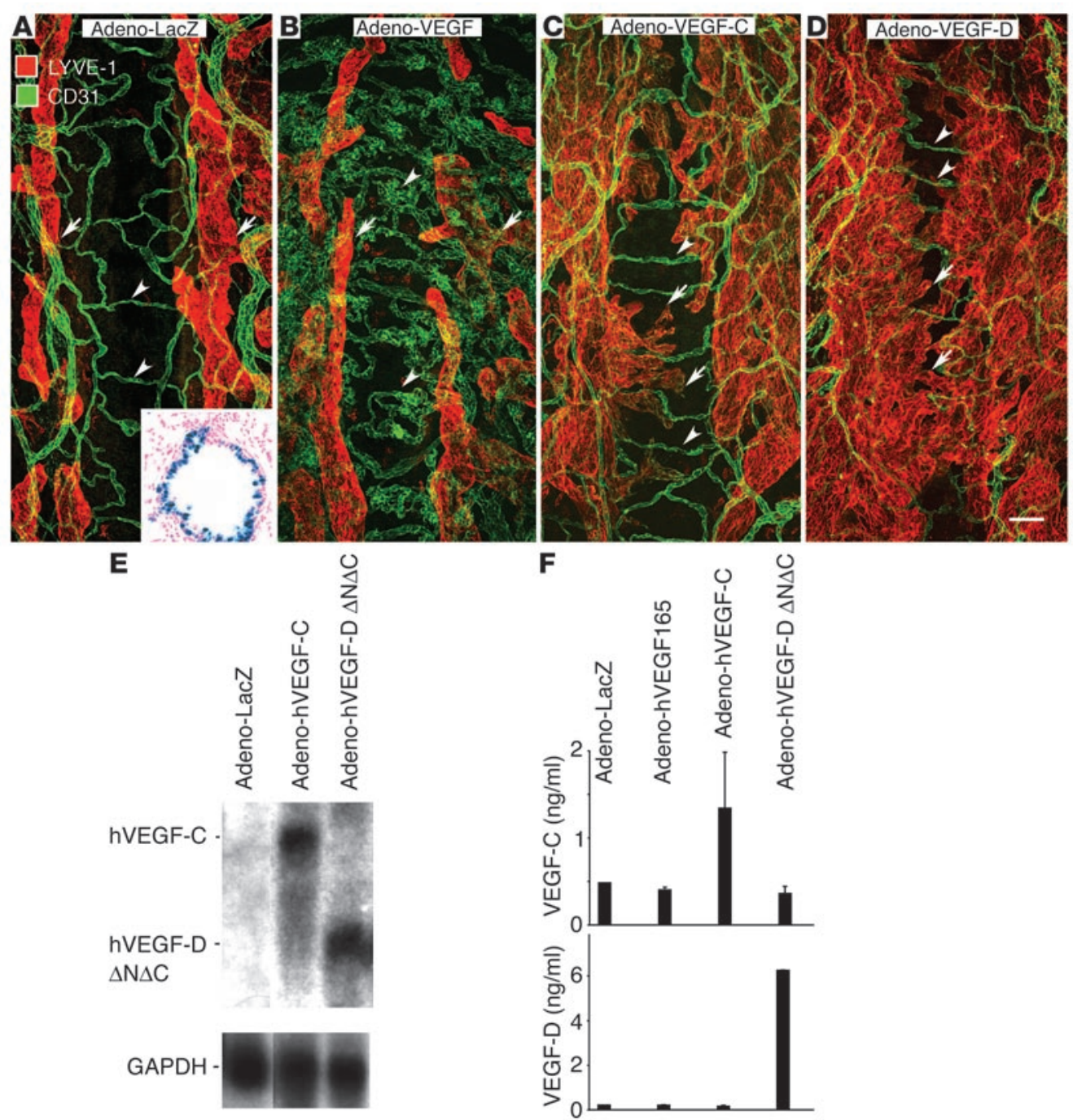

Figure 3

Overexpression of VEGF family growth factors by adenoviral vectors. Tracheal lymphatic vessels (red) and blood vessels (green) 10 days after intranasal inoculation of nude mice with adenoviral vectors. (A) Transduction of airway epithelial cells by adenovirus encoding LacZ (Adeno-LacZ), shown by X-gal staining (inset, blue), had no apparent effect on lymphatic vessels (arrows) or blood vessels (arrowheads). (B) Adenoviral VEGF induced angiogenesis (arrowheads) but no detectable lymphangiogenesis (arrows). (C) Adenoviral VEGF-C induced extensive lymphangiogenesis (arrows) but no angiogenesis (arrowheads). (D) Adenoviral VEGF-D induced widespread lymphangiogenesis but no apparent change in blood vessels (arrowheads); lymphatic vessels appear to coalesce (arrows). Scale bar in D (100 $\mu \mathrm{m})$ applies to all figures. (E) Northern blot showing human VEGF-C (hVEGF-C) and VEGF-D mRNA in extracts of lungs 10 days after inoculation with adenoviral LacZ, human VEGF-C, or human VEGF-D $\triangle N \Delta C$. (F) Results of ELISA showing VEGF-C and VEGF-D protein in lungs 10 days after inoculation with adenoviral LacZ, human VEGF165, VEGF-C, or VEGF-D $\Delta \mathrm{N} \Delta \mathrm{C}$.

dividing endothelial cells were within $20 \mu \mathrm{m}$ of the tip of lymphatic sprouts (Figure 1J). Another 10\% were in large collecting lymphatic vessels (mean diameter $130 \mu \mathrm{m}$; Figure $1 \mathrm{~K}$ ).

VEGFR-3 signaling is required for lymphangiogenesis in inflamed airways. We used multiple strategies to identify the growth factor signaling systems that drive angiogenesis and lymphangiogenesis in chronically inflamed airways. In the first step, we determined whether receptors for VEGF family members were expressed on airway blood vessels or lymphatic vessels. VEGFR-2 immunoreactivity was strong on blood capillaries, moderate on lymphatic vessels, and weak on arterioles and venules (Figure 2A). VEGFR-3 immunoreactivity was absent on blood vessels but strong on lymphatic vessels (Figure 2B). These results suggest that VEGFR-2 signaling might drive angiogenesis but either VEGFR-2 or VEGFR-3 could mediate lymphangiogenesis.

Because VEGFR-3 signaling is essential for lymphangiogenesis in some conditions $(4,21)$, we sought to determine whether the pathway is important for lymphangiogenesis in M. pulmonis-infected mice. Treatment with soluble VEGFR-3-Ig, which blocks VEGFR-3 signaling by binding VEGF-C and VEGF-D, almost completely prevented lymphatic growth during the first week of infection (Figure 2, C and D). Measurements showed a 96\% reduction in the number of lymphatic sprouts (Figure 2E). No impairment in blood vessel remodeling or growth was evident after treatment with soluble VEGFR-3-Ig.

In another strategy of blocking VEGF receptor signaling, mice were treated with function-blocking monoclonal antibodies against VEGFR-1, VEGFR-2, or VEGFR-3 at doses reported to be effective in other mouse models (22-24). Treatment with antibodies against VEGFR-1 (Figure 2F) or VEGFR-2 had no effect on growth of lymphatic vessels after M. pulmonis infection, but the anti-VEGFR-3 antibody almost totally blocked lymphangiogenesis during 2 weeks of infection (Figure 2G). Measurements showed an $87 \%$ reduction in lymphatic growth after VEGFR-3 antibody treatment but no significant change in lymphatic vessels after VEGFR-1 or VEGFR-2 antibody treatment (Figure 1H). A combination of antibodies against VEGFR-2 and VEGFR-3 had an effect no greater than anti-VEGFR-3 alone (Figure $1 \mathrm{H})$. None of these antibodies reduced growth or enlargement of airway blood vessels after infection (Figure $2 \mathrm{H}$ ).

VEGF-C and VEGF-D drive lymphangiogenesis in airways. To address the role of VEGFR-3 ligands in lymphangiogenesis in the respiratory tract, we used an adenoviral delivery system to generate VEGF-C or 


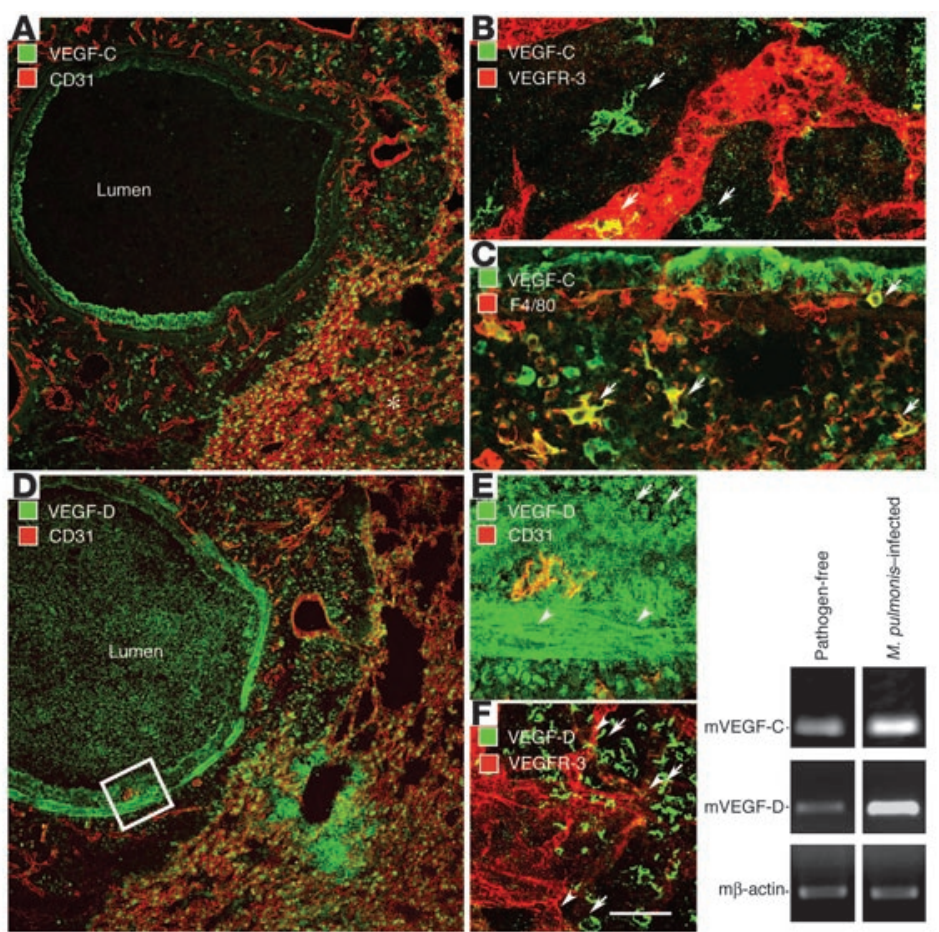

\section{Figure 4}

VEGF-C and VEGF-D in M. pulmonis-infected airways. Immunohistochemical staining of VEGF-C (A-C) and VEGF-D (D-F) in mouse airways and lung 14 days after infection. (A) VEGF-C (green) in epithelium, peribronchial inflammatory cells, and type II alveolar epithelial cells (asterisks) of lung. Blood vessels stained for CD31 (red). (B) VEGF-C (green) in inflammatory cells (arrows) near sprouting lymphatic vessels (red) in tracheal whole mount. (C) Colocalization of VEGF-C immunoreactivity (green) and F4/80 immunoreactivity (red) in macrophages (yellow, arrows). (D) Strong VEGF-D staining (green) of neutrophils in airway lumen. (E) VEGF-D-positive neutrophils (boxed area in $\mathbf{D}$ ) shown at higher magnification in airway lumen (arrows, green) and airway smooth muscle cells (arrowheads). Blood vessels stained for CD31 (red). (F) VEGF-D immunoreactivity in neutrophils (arrows, green) in tracheal mucosa near sprouting lymphatic vessels (arrowheads) stained for VEGFR-3 (red). Scale bar in $\mathbf{F}$ applies to all figures: $200 \mu \mathrm{m}$ in $\mathbf{A}$ and $\mathbf{D}$ and $50 \mu \mathrm{m}$ in B, C, E, and $\mathbf{F}$. (G) Results of RT-PCR analysis showing higher expression of mouse mRNA for VEGF-C (mVEGF-C) and VEGF-D in tracheas after 14 days of infection.
VEGF-D in airway epithelial cells. VEGF was generated in the same way for comparison. All 3 of these growth factors have been reported to induce angiogenesis and/or lymphangiogenesis (6, 8-10).

Mice were inoculated intranasally with LacZ-expressing adenovirus to determine whether transduction of airway epithelial cells by itself had effects on blood vessels or lymphatic vessels. Airway epithelial cells of these mice had strong $\beta$-gal expression (Figure $3 \mathrm{~A}$, inset) but no change in blood or lymphatic vasculature (Figure $3 \mathrm{~A})$. In contrast, inoculation with VEGF adenovirus induced prolific sprouting and growth of blood vessels but no lymphangiogenesis (Figure 3B). Conversely, inoculation with VEGF-C adenovirus induced growth of lymphatic filopodia and sprouts similar to those seen in M.pulmonisinfected mice but did not cause detectable changes in blood vessels (Figure 3C). Adenoviral processed VEGF-D truncated at $\mathrm{N}$ and $\mathrm{C}$ termini (VEGF-D $\Delta \mathrm{N} \Delta \mathrm{C}$ ), the mature form of VEGF-D (25), induced the formation of giant sheet-like lymphatic vessels in the trachea (Figure 3D) and twice as many lymphatic sprouts as did VEGF-C ( $30 \pm 4$ vs. $15 \pm 2$ sprouts $/ \mathrm{mm}^{2}$ of mucosa; $P<0.05$ ). However, VEGF-D $\Delta \mathrm{N} \Delta \mathrm{C}$, which stimulates angiogenesis in skeletal muscle (6), had no apparent effect on airway blood vessels (Figure 3D).

Inoculation of mice with VEGF-C or VEGF-D adenovirus conspicuously increased the corresponding mRNA in Northern blots of lung (Figure 3E). VEGF-C protein was increased approximately 3-fold, and VEGF-D was increased 30-fold above baseline amounts, as determined by ELISA (Figure $3 \mathrm{~F}$ ).

Cellular sources of VEGF-C and VEGF-D in inflamed airways. Inflammatory cells were rare in the airways of pathogen-free mice but were abundant after $M$. pulmonis infection. Neutrophils were not detected in the airway mucosa of pathogen-free $\mathrm{C} 3 \mathrm{H}$ mice but were abundant after infection, reaching a peak at 7 days $\left(1,581 \pm 332\right.$ neutrophils $\left./ \mathrm{mm}^{2}\right)$. Neutrophils were also abundant in the airway lumen. The massive influx of neutrophils was followed by a smaller, more gradual influx of macrophages and lymphocytes into the mucosa but not the lumen.
In the respiratory tract of pathogen-free mice, most VEGF-C immunoreactivity was in airway epithelial cells and surfactantproducing type II alveolar epithelial cells of the lung (data not shown). However, after infection, VEGF-C immunoreactivity was strong in mucosal inflammatory cells as well as in airway epithelial cells (Figure 4, A and B). In addition, some F4/80-positive macrophages had VEGF-C immunoreactivity (Figure 4C). VEGF-D immunoreactivity was minimal in the airways of pathogen-free mice but, after M. pulmonis infection, was strong in neutrophils in the mucosa and lumen (Figure 4, D-F). VEGF-D staining was also strong in macrophages, airway smooth muscle cells, and focal regions of some lymphatic vessels. In addition, staining for both VEGF-C and VEGF-D was diffuse and weak in basement membrane (Figure 4E). No staining was present when the primary antibody was omitted or replaced by preimmune serum (data not shown). Consistent with these findings, mRNAs for VEGF-C and VEGF-D increased in the airways after $M$. pulmonis infection, according to semiquantitative RT-PCR (Figure 4G).

Blocking lymphangiogenesis impairs lymphatic function and promotes mucosal edema. The amount of plasma leakage in the airway mucosa increases after $M$. pulmonis infection because the new and remodeled blood vessels are leaky and have increased surface area (17). The blood vessels are also enlarged and unusually sensitive to inflammatory mediators (17). Expansion of the lymphatic network after infection should accommodate the increased flux of tissue fluid. We therefore asked whether failure of lymphangiogenesis after infection impaired fluid drainage and immune cell transport to local lymph nodes and promoted bronchial lymphedema. Using Evans blue dye accumulation as a measure of leakage, we found that the amount of extravasated dye increased significantly after infection and increased further when lymphangiogenesis was inhibited more than $90 \%$ by systemic treatment with soluble VEGFR-3-Ig delivered by adenovirus prior to infection (Figure 5A). 
A
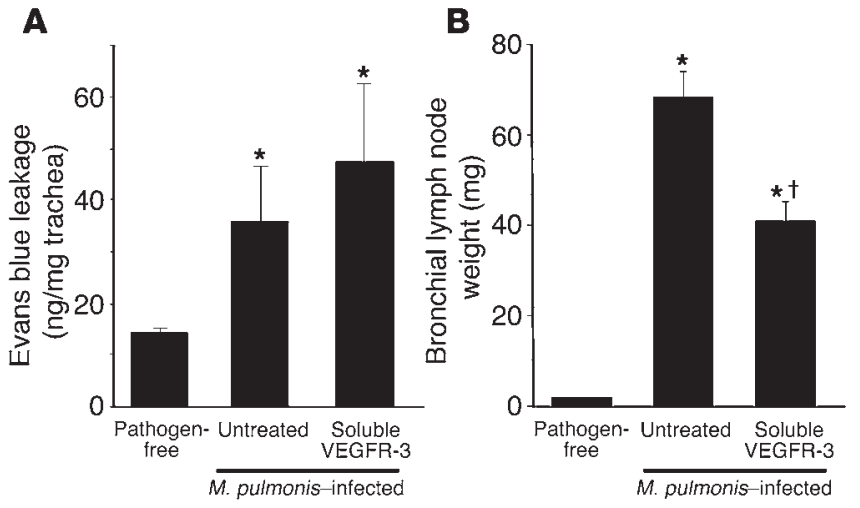

Figure 5

Consequences of blocking lymphangiogenesis in inflamed airways. Comparison of 3 groups of $\mathrm{C} 3 \mathrm{H}$ mice: pathogen-free, infected with M. pulmonis for 14 days, and infected with lymphangiogenesis blocked by concurrent treatment with soluble VEGFR-3-Ig delivered by adenovirus. (A) Evans blue accumulation in trachea over $30 \mathrm{~min}-$ utes showing significantly increased dye accumulation after infection $\left({ }^{\star} P<0.05\right)$ but even greater accumulation when lymphangiogenesis was blocked by soluble VEGFR-3-Ig. (B) Lymph nodes were enlarged after infection $\left({ }^{*} P<0.05\right)$, but significantly less so when lymphangiogenesis was inhibited by soluble VEGFR-3-Ig $\left({ }^{\dagger} P<0.05\right)$.

Bronchial lymph nodes that receive drainage from the airways and lungs increase in weight with time after M. pulmonis infection (18), a finding consistent with the proliferation of lymphocytes triggered by activated antigen-presenting cells and superantigens or mitogens from the organisms (26). Soluble VEGFR-3-Ig treatment prior to infection diminished this response, as reflected by an approximately $40 \%$ reduction in bronchial lymph node enlargement from the usual 20- to 30 -fold increase after 2 weeks of infection (Figure 5B).

The weights of bronchial lymph nodes and lungs turned out to be useful indicators of disease severity. Lung weight increased 2- or 3 -fold during the infection, as leukocytes and fluid accumulated, but was significantly lower after 2 weeks of antibiotic treatment and essentially normal by 6 weeks of treatment (Figure 6A). Bronchial lymph nodes decreased in size more slowly during treatment. There was no change after 2 weeks of treatment, but after 12 weeks, bronchial lymph nodes lost $88 \%$ of the enlargement present at the onset of treatment (Figure 6A).

Persistence of hyperplastic lymphatic vessels during resolution of inflammation. Treatment of M. pulmonis infection with antibiotics or steroids rapidly and fully reverses the remodeling and proliferation of blood vessels in the airway mucosa $(13,14)$. Consistent with those reports, the present experiments confirmed that remodeled blood vessels returned to their normal state within 4 weeks of antibiotic treatment (Figure 6, B-D). Lymphatic sprouting stopped during treatment, but the extensive network of new lymphatic vessels did not show a corresponding decrease even after 12 weeks of treatment.

Some normalization of lymphatic vessels was evident after 2 and 4 weeks of treatment with antibiotic. Amounts of lymphatic filopodia were reduced by $70 \%$ and $95 \%$ at 2 and 4 weeks of treatment with antibiotic, respectively, compared with the number present at the onset of treatment at 2 weeks of infection (Figure 6B). With this change, lymphatic vessels acquired smoother contours. In addition, some lymphatic vessels were thinner or had constrictions along their length. Seemingly isolated segments of LYVE-1- positive lymphatic vessel were also present. The overall intensity of LYVE-1 immunoreactivity of lymphatic sprouts was reduced after treatment but varied in individual lymphatic endothelial cells from intense to almost absent (Figure 6, C and D). Immunoreactivity to LYVE-1 was weaker than immunoreactivity to CD31 in some lymphatic vessels (Figure 6D).

Compared to the large reduction in sprouts, measurements of the overall extent of the lymphatic network changed relatively little over 12 weeks of treatment (Figure 6B). The pattern suggested a small decrease during the first 6 weeks of treatment followed by a plateau with little change thereafter, but the changes were not statistically significant (Figure 6B).

\section{Discussion}

The present study revealed that airway inflammation is accompanied not only by angiogenesis but also by extensive growth of lymphatic vessels. In mice with $M$. pulmonis infection, lymphangiogenesis appears to be driven by VEGF-C and VEGF-D from inflammatory cells that migrate into the airways. Adenoviral delivery of either of these growth factors induced lymphangiogenesis but not angiogenesis in the airways. Even the mature form of VEGF-D, which in humans binds to VEGFR-2 as well as VEGFR-3 $(6,25)$, induced abundant lymphangiogenesis without apparent angiogenic activity. After infection, expression of VEGF-C and VEGF-D was greatest in airway leukocytes. Inhibition of growth factor signaling through VEGFR-3 entirely blocked lymphatic sprouting after infection but had no apparent effect on blood vessel remodeling. Inhibition of lymphangiogenesis predisposed to bronchial lymphedema and reduced the infection-induced hypertrophy of draining lymph nodes. Newly formed lymphatic vessels - unlike the remodeled blood vessels - did not regress after the infection was treated.

New tools for studyinglymphangiogenesis. With the advent of new markers for and reagents that can be used to manipulate lymphatic vessels, the findings of many classical studies are beginning to be understood on a molecular level $(21,27,28)$. Earlier studies showed that lymphatic vessels proliferate in wounds and inflammation of skin $(3,16,29)$ and cornea $(30)$. Some experiments also suggested that lymphangiogenesis occurs more slowly than angiogenesis $(15,16,31)$. More recent studies show that lymphangiogenesis can be stimulated or inhibited by agonists or antagonists of VEGFR-3 signaling independent of angiogenesis $(32,33)$. Lymphatic vessels proliferate in inflammatory bowel disease, renal fibrosis, and transplant rejection $(34,35)$, but few models have been available for mechanistic studies of lymphangiogenesis under pathological conditions.

The present study revealed attributes of $M$. pulmonis infection of the respiratory tract in mice as a model for studying lymphangiogenesis in chronic inflammation. These features complement the model's attributes for studying vascular remodeling and angiogenesis $(18,19)$. The simple, orderly arrangement of blood vessels and lymphatic vessels in mouse airways coupled with the experimental conditions for examining the step-by-step progression toward chronic disease make it possible to dissect the cellular and molecular mechanisms of angiogenesis and lymphangiogenesis and their reversibility. Because the anatomical nature of the mouse trachea lends itself to the preparation of tissue whole mounts, blood vessels, lymphatic vessels, and inflammatory cells can be viewed simultaneously in three dimensions by fluorescence and confocal microscopy.

In lymphatic vessels that grow after M. pulmonis infection, sites of endothelial cell proliferation and vessel enlargement fit with a recent model of blood vessel growth (20). Delicate filopodia on 

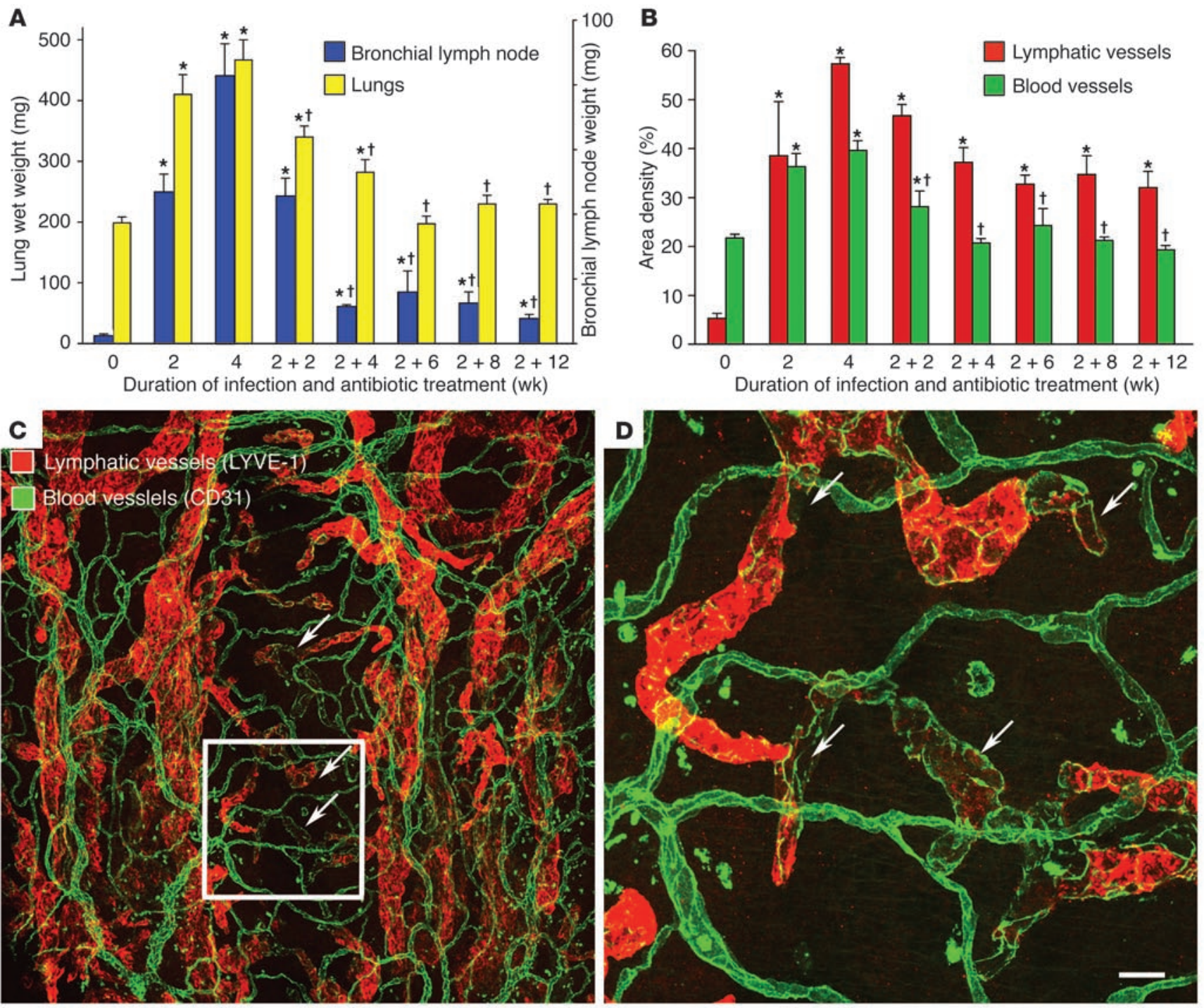

Figure 6

Persistence of new lymphatic vessels after treatment. (A and B) Weight of lungs and bronchial lymph nodes $(\mathbf{A})$ and abundance of tracheal lymphatic vessels and blood vessels (B) in pathogen-free mice (0), mice infected with M. pulmonis for 2 or 4 weeks (2 and 4$)$, and mice infected for 2 weeks and then treated with oxytetracycline for 2 to 12 weeks $(2+2$ through $2+12)$. The weight of both organs increased after infection and decreased toward normal after treatment. Airway blood vessels (green) showed a similar pattern, whereas lymphatic vessels (red) proliferated after infection but regressed little, even after 12 weeks of treatment. ${ }^{*} P<0.05$ vs. pathogen-free group; ${ }^{\dagger} P<0.05$ vs. 2 -week-infected group without treatment. (C and D) Confocal micrographs showing tracheal lymphatic vessels (red) and blood vessels (green) after infection for 2 weeks and then oxytetracycline treatment for 8 weeks. Blood vessels regressed almost to the pathogen-free state. Lymphatic vessels showed some changes but regressed only slightly; lymphatic vessels with constrictions and no LYVE-1 immunoreactivity in some cells are indicated by arrows. (D) Enlargement of the boxed area in C. Scale bar in D applies to both figures: $100 \mu \mathrm{m}$ in $\mathbf{C}, 25 \mu \mathrm{m}$ in $\mathbf{D}$.

lymphatic endothelial cells at the tips of sprouts marked sites of vessel growth, but cell division was most common in the stalk, one or two cells behind the tip. Enlargement of large collecting lymphatic vessels apparently resulted from proliferation of endothelial cells combined with distention by lymph.

Role of VEGFR-3 signaling. In our experiments, we used 2 approaches to block VEGFR-3 signaling, with the goal of determining whether lymphangiogenesis in the M. pulmonis model of chronic inflammation is driven by VEGFR-3 signaling, as it is in some other systems $(4,21)$. Both approaches - using soluble VEGFR-3-Ig and using function-blocking antibody - almost completely blocked lymphatic growth. By comparison, inhibition of VEGFR-1 or VEGFR-2 had no apparent effect on lymphatic growth, and inhibition of VEGFR-2 did not augment the action of VEGFR-3 blockade.

Next we found that either VEGF-C or VEGF-D triggered intense lymphangiogenesis in the airways when delivered by adenoviral vector. VEGF stimulated angiogenesis but not lymphangiogenesis, despite some expression of VEGFR-2 on endothelial cells of lymphatic vessels. Together, these findings are consistent with the identification of VEGFR-3 signaling as the principal driving force for lymphatic growth in this model. VEGF and VEGFR-2 signaling play little or no apparent role. 


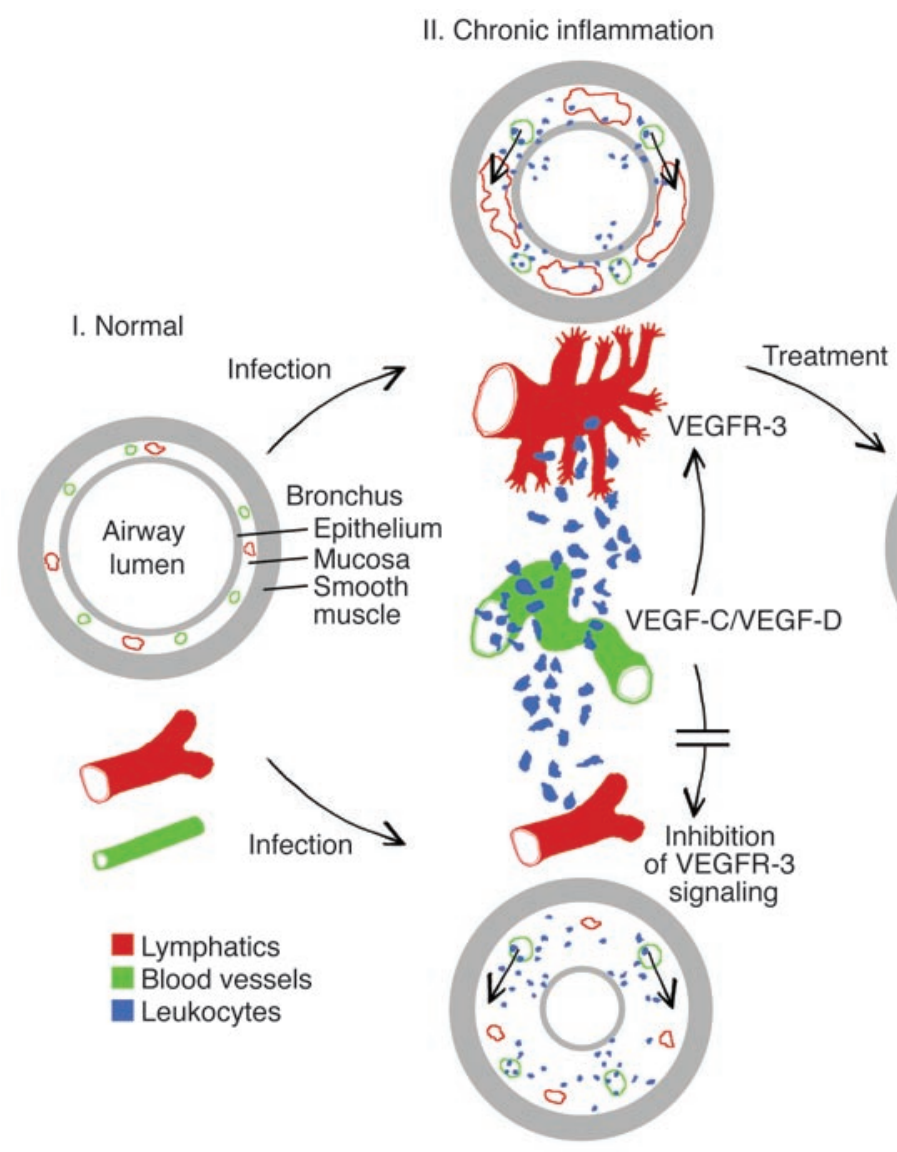

III. Bronchial lymphedema

\section{Figure 7}

Role of lymphangiogenesis in airway inflammation. The diagram shows relationships of changes in lymphatic vessels (red), blood vessels (green), inflammatory cells (blue), and plasma leakage (short arrows) in inflamed airway mucosa. Cross-sections of bronchi and vessel schematics compare 4 conditions. (I) In normal airways, blood vessels have little or no leukocyte traffic; baseline leakage drains via lymphatic vessels. (II) After infection, activated antigenpresenting cells traffic from airways to local lymph nodes, evoking an immune response. Mucosal capillaries remodel into activated venules that mediate leukocyte influx. These cells release VEGF-C and VEGF-D, which drive lymphangiogenesis via VEGFR-3 signaling in lymphatic endothelial cells. The lymphatic network expands by sprouting from existing lymphatic vessels to accommodate the increased leakage from venules and increased trafficking of immune cells to lymph nodes. (III) If lymphangiogenesis does not occur, leakage exceeds drainage, bronchial lymphedema develops, trafficking of immune cells to lymph nodes decreases, and the immune response is reduced. (IV) Treatment decreases the inflammatory stimulus, allowing blood vessels to return to the baseline state. Leukocyte influx decreases, and the stimulus for lymphangiogenesis diminishes, but lymphatic vessels that formed during the infection persist - ready for the next infection.
The signaling system that drives angiogenesis after M. pulmonis infection is unknown. Antibodies that block the function of VEGFR-1 or VEGFR-2 did not significantly reduce blood vessel remodeling, which indicates that VEGF, VEGF-B, and placental growth factor (PlGF) are not responsible for changes in these vessels. Similarly, neither VEGF-C nor VEGF-D stimulated angiogenesis in the model, yet both can stimulate angiogenesis under certain conditions $(5,6,8,9)$. Local concentration and not overall dose of growth factors determine the exact pattern of vessel remodeling (36). Even the mature form of VEGF-D, which can induce tyrosine phosphorylation of VEGFR-2 $(6,9)$, produced only lymphangiogenesis in our model.

Sources of VEGF-C and VEGF-D in inflamed airways. Cells in the airways of infected mice had significantly greater expression of VEGF-C and VEGF-D, as reflected by mRNA analysis and immunohistochemistry. Localization studies revealed that VEGF-C was expressed by mononuclear inflammatory cells that were not present in pathogen-free mice. Some of these cells, likely macrophages or dendritic cells, were close to growing lymphatic vessels. Mononuclear cells are a known source of VEGF-C $(33,35,37)$. VEGF-C was also expressed in airway epithelial cells, both with and without infection. VEGF-D was expressed in neutrophils in the mucosa and lumen of inflamed airways and in other inflammatory cells. Little VEGF-D expression was detected in airways or lung of pathogen-free mice, consistent with previous findings (25).

Dendritic cells, as antigen-presenting cells activated by bacterial antigens, migrate via lymphatic vessels to local lymph nodes within 24 hours of infection to initiate an immune response, with the proliferation of lymphocytes causing hypertrophy of the lymph nodes (38). Activated dendritic cells, which express VEGF-C (37), may help to drive the development of the lymphatic network they use as a route to lymph nodes. In inflammation, dendritic cells are believed to migrate toward lymphatic vessels along a chemotactic gradient of mediators, including chemokines CCL19 and CCL21 from lymphatic endothelial cells $(39,40)$. Dendritic cells express CCR7 receptors for these ligands $(39,40)$. Thus, dendritic cells and lymphatic vessels express ligands and receptors for one another that may act in a reciprocal fashion.

The full range of factors that activate dendritic cells is unclear, but IL- $1 \beta$ and TNF- $\alpha$ are both present after $M$. pulmonis infection (41) and can increase VEGF-C expression (42). Although many of the details are still missing, it appears that key cells, mediators, and feedback loops necessary to induce lymphangiogenesis are present in the airways after M. pulmonis infection (Figure 7).

Functional consequences of impaired lymphangiogenesis in airways. Results of the present study suggest that inhibition of VEGFR-3 signaling reduces the transport of fluid and antigen-presenting cells from airways to lymph nodes via lymphatic vessels, thereby exaggerating mucosal edema and dampening the afferent limb of the immune response. Consistent with these consequences of impaired lymphangiogenesis is the finding that blocking lymphatic transport from inflamed rabbit ears may exacerbate local edema and eventually lead to necrosis (43). Similarly, inhibition of VEGFR-3 signaling suppresses dendritic cell trafficking from the cornea to draining lymph nodes and corneal transplant rejection (44). 
Additional experiments will be needed to develop a more complete understanding of the net effect of these actions on the severity of inflammatory responses.

Angiogenesis and mucosal swelling are part of the pathophysiology of chronic inflammatory airway diseases such as asthma and chronic bronchitis $(2,45)$. Like other causes of sustained airway inflammation, mycoplasmal infection in rodents is accompanied by inflammatory cell influx, angiogenesis, mucosal edema, epithelial changes, and fibrosis (17). Mycoplasma pneumoniae infection also results in bronchial hyperreactivity but not necessarily all the features of asthma $(46,47)$.

To our knowledge, the involvement of lymphatic abnormalities in bronchial hyperreactivity or airflow obstruction has not been examined. Impaired drainage of edema fluid would be expected to lead to airway wall thickening, reduced wall compliance, and narrowing of the airway lumen (Figure 7). If lymphangiogenesis is impaired in inflamed airways, restoration of lymphangiogenesis could complement conventional therapies for inflammatory airway disease.

Persistence of lymphatic vessels after treatment. Although additional growth of lymphatic vessels induced by $M$. pulmonis infection was readily prevented by treatment with antibiotic, once formed, the new lymphatic vessels persisted after the inflammation resolved. The relative stability of lymphatic vessels under these conditions clearly differs from the corresponding changes in blood vessels, which rapidly regressed after antibiotic treatment. Because lymphatic vessels lack pericytes and basement membrane $(16,30)$, their resilience is at odds with current dogma that such features protect endothelial cells from regression (48). Even though most lymphatic vessels did not regress, some had markedly reduced LYVE-1 expression. Downregulation of LYVE-1 is also observed in lymphatic vessels of some tumors and acute skin inflammation (L. Johnson and D.G. Jackson, unpublished data), which suggests that expression is responsive to tissue microenvironment.

In conclusion, lymphangiogenesis begins slowly in airway inflammation but, after a few weeks, overtakes the remodeling and proliferation of blood vessels and persists after the inflammation resolves. Lymphangiogenesis in inflamed airways is mediated by VEGF-C and VEGF-D derived mainly from inflammatory cells via VEGFR-3 signaling in lymphatic endothelial cells. The expanded lymphatic network provides conduits for extravasated fluid from leaky blood vessels and activated antigen-presenting cells to local lymph nodes. Impaired lymphangiogenesis in airway inflammation may interfere with fluid clearance and lead to bronchial lymphedema. A better understanding of the factors that stimulate or inhibit lymphatic growth or accelerate the regression of new lymphatic vessels may offer new targets for intervention in the treatment of asthma and other inflammatory airway diseases.

\section{Methods}

Animals. Specific pathogen-free $\mathrm{C} 3 \mathrm{H}$ or C57BL/6 mice (Charles River Laboratories Inc.) at 8 weeks of age were used for M. pulmonis infection experiments, and immunodeficient NMRI nu/nu mice (Harlan) were used to study adenoviral transduction of airway epithelial cells in vivo. Mice were housed under barrier conditions, and sentinels were routinely tested serologically for common pathogens. Before being subject to experimental procedures, mice were anesthetized with ketamine $(100 \mathrm{mg} / \mathrm{kg})$ and xylazine $(10 \mathrm{mg} / \mathrm{kg})$ injected intramuscularly. The Institutional Animal Care and Use Committees of the UCSF and the University of Helsinki approved all procedures.
M. pulmonis infection and antibiotic treatment. $\mathrm{C} 3 \mathrm{H}$ or $\mathrm{C} 57 \mathrm{BL} / 6$ mice were inoculated intranasally on day 0 with $50 \mu \mathrm{l}$ of broth containing $10^{5}$ or $10^{6} \mathrm{CFU}$, respectively, of $M$. pulmonis organisms (strain CT7; University of Alabama at Birmingham) (19). In reversal experiments, infected $\mathrm{C} 3 \mathrm{H}$ mice were injected subcutaneously once daily with oxytetracycline $(20 \mathrm{mg} / \mathrm{kg}$; Phoenix Pharmaceuticals Inc.) starting on day 14 of infection and continuing for 2-12 weeks (13). At the end of the experiment, mice were anesthetized and perfused with fixative; trachea, lungs, and bronchial lymph nodes were removed.

Inhibition of VEGF receptor signaling. Soluble VEGFR-3 was used to block VEGFR-3 signaling. This decoy receptor was produced systemically by adenoviral delivery of soluble chimeric VEGFR-3 coupled to a mouse Ig fragment (49). Adenovirus $\left(1 \times 10^{9} \mathrm{PFU}\right.$ in $\left.100 \mu \mathrm{l}\right)$ was injected i.v. into $\mathrm{C} 3 \mathrm{H}$ mice on the day of infection. Lymphangiogenesis and angiogenesis were assessed 7 or 14 days later. To prepare the vector, we produced cDNA encoding the first 3 Ig loops of mouse VEGFR-3 (GenBank accession number NM008029.1, nucleotides $117-1053$ ) by RT-PCR using the primers 5 '-GCGCAAGCTTGTACTCCATGACCCCTCCAAC-3' and 5'-GAGCGATATCCAGGACAGGTCCTTTGAGCCA-3' and total mouse liver RNA as a template. The PCR product and mouse $\mathrm{Fc}$ fragment from the plasmid $\mathrm{mFc} /$ plasmid Bluescript were cloned into pSecTag expression vector (Invitrogen Corp.), and a fragment containing both mVEGFR-3 and Fc domains was further subcloned into PAdCMV vector for production of recombinant adenoviruses (50).

Alternatively, signaling by VEGFR-1, VEGFR-2, or VEGFR-3 was inhibited using selective function-blocking rat monoclonal antibodies from clones MF1, DC101, and 31C1, respectively, at doses known to be effective (ImClone Systems Inc.; refs. 22-24). Rat IgG was used as a control. Antibodies were injected i.p. in $\mathrm{C} 3 \mathrm{H}$ mice at an initial dose of $2 \mathrm{mg} /$ mouse on the day of infection followed every other day with doses of $0.8 \mathrm{mg} / \mathrm{mouse}$ for 14 days.

Adenoviral transduction of airway epithelium with growth factors. Airway epithelial cells were transduced with genes encoding VEGF, VEGF-C, VEGF-D, or LacZ by intranasal inoculation of nude mice with adenovirus at 5 to approximately $10 \times 10^{8} \mathrm{PFU}$ in $50 \mu$ l. Adenovirus expressing full-length cDNA for human VEGF165, VEGF-C, or VEGF-D $\Delta \mathrm{N} \Delta \mathrm{C}$ (6), or a nuclear-targeted LacZ gene as an inactive control, was prepared as previously described $(8,51)$. Each adenovirus was analyzed to ensure absence of replication-competent virus, lipopolysaccharide, mycoplasma, or other contaminants. Lymphangiogenesis and angiogenesis were assessed 10 days after inoculation. Transduction of epithelial cells was verified by X-gal staining (7) or $\beta$-gal immunoreactivity after inoculation with the LacZ adenovirus. Expression of VEGF, VEGF-C, and VEGF-D was verified by Northern blotting of RNA extracted from lungs and tracheas.

Immunohistochemistry. The vasculature of anesthetized mice was perfused for 2 minutes with 1\% paraformaldehyde in PBS, pH 7.4 (52). Trachea, lung, and liver were removed and immersed in the fixative for 1 hour. Tracheas were stained as whole mounts and lung and liver as $20-\mu \mathrm{m}$ cryostat sections with 1 or more primary antibodies (52): (a) lymphatic vessels: rabbit polyclonal antibody against LYVE-1, 1:1000 (D.G. Jackson laboratory) (53); (b) blood vessels: monoclonal antibodies against mouse CD31, hamster clone $2 \mathrm{H8}, 1: 500$, Chemicon International Inc. or rat clone MEC 13.3, 1:500 (BD Biosciences - Pharmingen); (c) VEGF-C: rabbit polyclonal antibody no. 6 to full-length human VEGF-C, 1:500; (d) VEGF-D: rabbit polyclonal antibody no. 490 to mouse VEGF-D, 1:500 (Herbert Weich, ReliaTech, Braunschweig, Germany); (e) VEGFR-2: rabbit polyclonal antibody, 1:2000 (Rolf Brekken, University of Texas Southwestern, Dallas, Texas, USA); (f) VEGFR-3: goat polyclonal antibody, 1:500 (R\&D Systems); (g) leukocytes: rat monoclonal antibody against CD45, 1:250 (BD Biosciences - Pharmingen; (h) macrophages: rat monoclonal antibody against F4/80, 1:500 (BD Biosciences - Pharmingen); (i) dividing cells: rabbit polyclonal antibody against phosphohistone $\mathrm{H} 3,1: 500$ (Upstate 
Biotechnology Inc.); (j) LacZ: rabbit polyclonal antibody against $\beta$-gal, 1:500 (Chemicon International Inc.). Species-specific secondary antibodies were labeled with Cy3, Cy5, or FITC (1:400; Jackson ImmunoResearch Laboratories Inc.). For control experiments, the primary antibody was omitted or the preimmune serum substituted. Specimens were examined with a Zeiss Axiophot microscope or Zeiss LSM-510 confocal microscope (52).

Dividing lymphatic endothelial cells. Dividing lymphatic endothelial cells were identified by colocalization of staining for phosphohistone H3 and VEGFR-3. Tracheal whole mounts were examined by fine focusing with an oil-immersion lens $(\times 40$; numerical aperture, 1.0 ; final screen magnification $\times 1,200)$ or in optical sections ( $0.7 \mu \mathrm{m}$ thick) obtained by confocal microscopy. The diameter of lymphatic vessels and distance of 20 labeled nuclei to the tip of lymphatic sprouts were measured in tracheas from 5 mice per group.

RT-PCR analysis. Tracheas and lungs of pathogen-free mice and mice infected with $M$. pulmonis for 14 days were removed and immersed in RNAlater (QIAGEN Inc.). Total RNA was extracted from tissues using the RNAeasy kit (QIAGEN Inc.). Genomic DNA was eradicated by treatment with DNAse (QIAGEN Inc.), and cDNA was generated using Omniscript reverse transcriptase (QIAGEN Inc.), oligo-dT primers, and $150 \mathrm{ng}$ of total RNA. Equal amounts of cDNA were subjected to $\mathrm{PCR}$ with primers specific for mouse Vegfc (7) or Vegfd (GenBank accession number NM010216, base pairs 903-1348) cDNA, and $\beta$-actin was used as a loading control.

Northern blots. RNA extracted as above was electrophoresed, transferred to a nylon membrane, and hybridized to radioactive probes for human VEGF-C (GenBank accession number NM005429.2, base pairs 80-2076) and VEGF-D $\triangle \mathrm{N} \Delta \mathrm{C}$ (GenBank accession number NM004469.2, base pairs 509-1572) (5). A GAPDH probe served as a loading control.

ELISA. VEGF-C and VEGF-D in the lungs of nude mice treated intranasally with adenoviruses encoding LacZ, VEGF165, VEGF-C, or VEGF-D $\triangle \mathrm{N} \triangle \mathrm{C} 10$ days previously were measured by ELISA (human VEGF-C ELISA [no. BMS297; Bender MedSystems], human VEGF-D ELISA [no. DVED00; R\&D Systems]). Full-length recombinant human VEGF-C and supernatant from HeLa cells transfected with adenoviral VEGF-D $\Delta N \Delta C$ were used as positive controls. The mean sensitivities of the ELISA tests were $11.4 \mathrm{pg} / \mathrm{ml}$ for VEGF-C and $12.8 \mathrm{pg} / \mathrm{ml}$ for VEGF-D.

Extravasation of Evans blue dye. Anesthetized mice received an i.v. injection of Evans blue dye ( $30 \mathrm{mg} / \mathrm{kg}$; Sigma-Aldrich) and 30 minutes later were perfused via the left ventricle with $1 \%$ paraformaldehyde in citrate buffer, $\mathrm{pH} 3.5$ (18). Tracheas were weighed, and the dye was extracted in $1 \mathrm{ml}$ formamide overnight at $60^{\circ} \mathrm{C}$ and measured by spectrophotometry at $610 \mathrm{~nm}$.

Morphometric measurements and statistics. Area densities (percentage of total tissue area) of LYVE-1-positive lymphatic vessels and CD31-positive blood vessels viewed in real-time fluorescent images of tracheal whole mounts were measured by stereological point counting of 10 regions per trachea, each $1.7 \mathrm{~mm}^{2}$ in area. Lymphatic sprouts, defined as tapered projections visible at a screen magnification of $\times 180$, were counted in 5 regions per trachea, each $1.5 \mathrm{~mm}^{2}$ in area. Filopodia arising from lymphatic sprouts were counted at a screen magnification of $\times 600$ in 5 regions per trachea, each $0.13 \mathrm{~mm}^{2}$ in area. Values were expressed per square millimeter of tracheal mucosa. Neutrophils were counted in sections stained with $\mathrm{H} \& \mathrm{E}$. Values presented are mean $\pm \mathrm{SEM}$; $n=4-8$ mice per group. Significance of differences between means was assessed by analysis of variance followed by the Dunn-Bonferroni test for multiple comparisons, with the level of significance set at $P<0.05$.

\section{Acknowledgments}

We thank Carolyn Woo and Ian Kasman (UCSF) for help with histology and confocal microscopy and Jenny Tornberg, Johannes Lyytikkä, and Tapio Tainola (University of Helsinki) for expert technical assistance with adenovirus preparation. Rolf Brekken (University of Texas Southwestern, Dallas, Texas, USA) and Herbert Weich (ReliaTech, Braunschweig, Germany) kindly provided antibodies against VEGFR-2 and VEGF-D, respectively. Kris Persaud and Yan Wu (ImClone) helped produce the VEGF receptor blocking antibodies. Gerhardt Christofori (University of Basel, Basel, Switzerland) kindly provided the plasmid $\mathrm{mFc} /$ plasmid Bluescript for the mouse Fc fragment. This work was supported in part by grants from the National Heart, Lung, and Blood Institute of the NIH (HL-24136 and HL-59157), the Sandler Foundation for Basic Research in Asthma, the Vascular Mapping Project, and Angelworks Foundation (to D. McDonald); grants from the Finnish Academy of Sciences (to S. Ylä-Herttuala and K. Alitalo); and grants from Biocentrum Helsinki, the Human Frontier Science Program (HFSP R6P0231/2001-M), the European Union (QLK3-CT-2002-02059 and LSHG-CT-2004-503573), and the NIH (HD-37243) (to K. Alitalo). T.V. Petrova was supported by a Research Fellowship from the Finnish Academy of Sciences. S.A. Stacker and M.G Achen were supported by Senior Research Fellowships from the Pharmacia Foundation and the National Health and Medical Research Council of Australia (NHMRC), respectively, and by an NHMRC Program Grant.

Received for publication May 3, 2004, and accepted in revised form November 30, 2004.

Address correspondence to: Donald M. McDonald, Department of Anatomy, Room S-1363, University of California, San Francisco, California 94143, USA. Phone: (415) 476-2118; Fax: (415) 476-4845; E-mail: dmcd@itsa.ucsf.edu.

Tuomas Tammela and Erin Ator contributed equally to this work.
1. Wiggs, B.R., Bosken, C., Pare, P.D., James, A., and Hogg, J.C. 1992. A model of airway narrowing in asthma and in chronic obstructive pulmonary disease. Am. Rev. Respir. Dis. 145:1251-1258.

2. Wilson, J. 2000. The bronchial microcirculation in asthma. Clin. Exp. Allergy. 30:51-53.

3. Pullinger, B.D., and Florey, H.W. 1937. Proliferation of lymphatics in inflammation. J. Pathol. Bact. 45:157-170.

4. Jussila, L., and Alitalo, K. 2002. Vascular growth factors and lymphangiogenesis. Physiol. Rev. 82:673-700.

5. Saaristo, A., et al. 2002. Adenoviral VEGF-C overexpression induces blood vessel enlargement, tortuosity, and leakiness but no sprouting angiogenesis in the skin or mucous membranes. FASEB J. 16:1041-1049.

6. Rissanen, T.T., et al. 2003. VEGF-D is the stron- gest angiogenic and lymphangiogenic effector among VEGFs delivered into skeletal muscle via adenoviruses. Circ. Res. 92:1098-1106.

7. Karkkainen, M.J., et al. 2004. Vascular endothelial growth factor $\mathrm{C}$ is required for sprouting of the first lymphatic vessels from embryonic veins. Nat. Immunol. 5:74-80.

8. Saaristo, A., et al. 2002. Lymphangiogenic gene therapy with minimal blood vascular side effects. J. Exp. Med. 196:719-730.

9. Byzova, T.V., et al. 2002. Adenovirus encoding vascular endothelial growth factor-D induces tissue-specific vascular patterns in vivo. Blood. 99:4434-4442.

10. Nagy, J.A., et al. 2002. Vascular permeability factor/vascular endothelial growth factor induces lymphangiogenesis as well as angiogenesis. J. Exp. Med. 196:1497-1506.

11. Cao, R., et al. 2004. PDGF-BB induces intratumor- al lymphangiogenesis and promotes lymphatic metastasis. Cancer Cell. 6:333-345.

12. Rockson, S.G. 2001. Lymphedema. Am. J. Med. 110:288-295.

13. Bowden, J.J., Schoeb, T.R., Lindsey, J.R., and McDonald, D.M. 1994. Dexamethasone and oxytetracycline reverse the potentiation of neurogenic inflammation in airways of rats with Mycoplasma pulmonis infection. Am. J. Respir. Crit. Care Med. 150:1391-1401.

14. Thurston, G., Maas, K., LaBarbara, A., and McDonald, D.M. 2000. Microvascular remodeling in chronic airway inflammation in mice. Clin. Exp. Pharm. Physiol. 27:836-841.

15. Clark, E., and Clark, E. 1937. Observations on living mammalian lymphatic capillaries-their relation to the blood vessels. Am. J. Anat. 60:253-298.

16. Yoffey, J., and Courtice, F. 1970. Lymphatics, lymph and 
the lymphomyeloid complex. Academic Press. New York, New York, USA/London, United Kingdom. 942 pp.

17. McDonald, D.M. 2001. Angiogenesis and remodeling of airway vasculature in chronic inflammation. Am. J. Respir. Crit. Care Med. 164:S39-S45.

18. Ezaki, T., et al. 2001. Time course of endothelial cell proliferation and microvascular remodeling in chronic inflammation. Am. J. Pathol. 158:2043-2055.

19. Thurston, G., Murphy, T.J., Baluk, P., Lindsey, J.R., and McDonald, D.M. 1998. Angiogenesis in mice with chronic airway inflammation: strain-dependent differences. Am. J. Pathol. 153:1099-1112.

20. Gerhardt, H., et al. 2003. VEGF guides angiogenic sprouting utilizing endothelial tip cell filopodia. J. Cell Biol. 161:1163-1177.

21. Lohela, M., Saaristo, A., Veikkola, T., and Alitalo, K. 2003. Lymphangiogenic growth factors, receptors and therapies. Thromb. Haemost. 90:167-184.

22. Luttun, A., et al. 2002. Revascularization of ischemic tissues by PlGF treatment, and inhibition of tumor angiogenesis, arthritis and atherosclerosis by anti-Flt1. Nat. Med. 8:831-840.

23. Prewett, M., et al. 1999. Antivascular endothelial growth factor receptor (fetal liver kinase 1) monoclonal antibody inhibits tumor angiogenesis and growth of several mouse and human tumors. Cancer Res. 59:5209-5218.

24. Pytowski, B., et al. 2005. Complete and specific inhibition of adult lymphatic regeneration by a novel VEGFR-3 neutralizing antibody. J. Natl. Cancer Inst. 97:14-21.

25. Stacker, S.A., et al. 1999. Biosynthesis of vascular endothelial growth factor-D involves proteolytic processing which generates non-covalent homodimers. J. Biol. Chem. 274:32127-32136.

26. Cartner, S.C., Simecka, J.W., Lindsey, J.R., Cassell, G.H., and Davis, J.K. 1995. Chronic respiratory mycoplasmosis in $\mathrm{C} 3 \mathrm{H} / \mathrm{HeN}$ and $\mathrm{C} 57 \mathrm{BL} / 6 \mathrm{~N}$ mice: lesion severity and antibody response. Infect. Immun. 63:4138-4142.

27. Oliver, G., and Detmar, M. 2002. The rediscovery of the lymphatic system: old and new insights into the development and biological function of the lymphatic vasculature. Genes Dev. 16:773-783.

28. Jackson, D.G. 2003. The lymphatics revisited: new perspectives from the hyaluronan receptor LYVE-1. Trends Cardiovasc. Med. 13:1-7.

29. Clark, E., and Clark, E. 1932. Observations on the new growth of lymphatic vessels as seen in trans- parent chambers introduced into the rabbit's ear. Am. J. Anat. 51:49-87.

30. Collin, H.B. 1971. The fine structure of growing corneal lymphatic vessels. J. Pathol. 104:99-113.

31. Junghans, B.M., and Collin, H.B. 1989. Limbal lymphangiogenesis after corneal injury: an autoradiographic study. Curr. Eye Res. 8:91-100.

32. Makinen, T., et al. 2001. Inhibition of lymphangiogenesis with resulting lymphedema in transgenic mice expressing soluble VEGF receptor-3. Nat. Med. 7:199-205.

33. Cursiefen, C., et al. 2004. VEGF-A stimulates lymphangiogenesis and hemangiogenesis in inflammatory neovascularization via macrophage recruitment. J. Clin. Invest. 113:1040-1050. doi:10.1172/JCI200420465.

34. Geleff, S., Schoppmann, S.F., and Oberhuber, G. 2003. Increase in podoplanin-expressing intestinal lymphatic vessels in inflammatory bowel disease. Virchows Arch. 442:231-237.

35. Kerjaschki, D., et al. 2004. Lymphatic neoangiogenesis in human kidney transplants is associated with immunologically active lymphocytic infiltrates. J. Am. Soc. Nephrol. 15:603-612.

36. Ozawa, C.R., et al. 2004. Microenvironmental VEGF concentration, not total dose, determines a threshold between normal and aberrant angiogenesis. J. Clin. Invest. 113:516-527. doi:10.1172/JCI200418420.

37. Hamrah, P., Chen, L., Zhang, Q., and Dana, M.R. 2003. Novel expression of vascular endothelial growth factor receptor (VEGFR)-3 and VEGF-C on corneal dendritic cells. Am. J. Pathol. 163:57-68.

38. Banchereau, J., and Steinman, R.M. 1998. Dendritic cells and the control of immunity. Nature. 392:245-252.

39. Randolph, G.J. 2001. Dendritic cell migration to lymph nodes: cytokines, chemokines, and lipid mediators. Semin. Immunol. 13:267-274.

40. Qu, P., Ji, R.C., and Kato, S. 2003. Histochemical analysis of lymphatic endothelial cells in the pancreas of non-obese diabetic mice. J. Anat. 203:523-530.

41. Faulkner, C.B., et al. 1995. Gene expression and production of tumor necrosis factor alpha, interleukin 1 , interleukin 6, and gamma interferon in $\mathrm{C} 3 \mathrm{H} / \mathrm{HeN}$ and $\mathrm{C} 57 \mathrm{BL} / 6 \mathrm{~N}$ mice in acute Mycoplasma pulmonis disease. Infect. Immun. 63:4084-4090.

42. Ristimaki, A., Narko, K., Enholm, B., Joukov, V., and Alitalo, K. 1998. Proinflammatory cytokines regulate expression of the lymphatic endothelial mitogen vascular endothelial growth factor-C. J. Biol. Chem. 273:8413-8418.

43. Casley-Smith, J.R., Clodius, L., and Foldi, M. 1977. Experimental blood vascular and lymphatic occlusion in the rabbit ear and the effect of benzopyrones. Arzneimittelforschung. 27:379-382.

44. Chen, L., et al. 2004. Vascular endothelial growth factor receptor- 3 mediates induction of corneal alloimmunity. Nat. Med. 10:813-815.

45. Hoshino, M., Nakamura, Y., and Hamid, Q.A. 2001. Gene expression of vascular endothelial growth factor and its receptors and angiogenesis in bronchial asthma. J. Allergy Clin. Immunol. 107:1034-1038.

46. Hardy, R.D., et al. 2001. Elevated cytokine and chemokine levels and prolonged pulmonary airflow resistance in a murine Mycoplasma pneumoniae pneumonia model: a microbiologic, histologic, immunologic, and respiratory plethysmographic profile. Infect. Immun. 69:3869-3876.

47. Chu, H.W., Honour, J.M., Rawlinson, C.A., Harbeck, R.J., and Martin, R.J. 2003. Effects of respiratory Mycoplasma pneumoniae infection on allergen-induced bronchial hyperresponsiveness and lung inflammation in mice. Infect. Immun. 71:1520-1526.

48. Benjamin, L.E. 2000. The controls of microvascular survival. Cancer Metastasis Rev. 19:75-81.

49. He, Y., et al. 2002. Suppression of tumor lymphangiogenesis and lymph node metastasis by blocking vascular endothelial growth factor receptor 3 signaling. J. Natl. Cancer Inst. 94:819-825.

50. Laitinen, M., et al. 1998. Adenovirus-mediated gene transfer to lower limb artery of patients with chronic critical leg ischemia. Hum. Gene Ther. 9:1481-1486.

51. Enholm, B., et al. 2001. Adenoviral expression of vascular endothelial growth factor-C induces lymphangiogenesis in the skin. Circ. Res. 88:623-629.

52. Inai, T., et al. 2004. Inhibition of vascular endothelial growth factor (VEGF) signaling in cancer causes loss of endothelial fenestrations, regression of tumor vessels, and appearance of basement membrane ghosts. Am. J. Pathol. 165:35-52.

53. Prevo, R., Banerji, S., Ferguson, D.J., Clasper, S., and Jackson, D.G. 2001. Mouse LYVE-1 is an endocytic receptor for hyaluronan in lymphatic endothelium. J. Biol. Chem. 276:19420-19430. 